\title{
Recent advances in molecular engineering of redox active organic molecules for nonaqueous flow batteries
}

\author{
Jeffrey A. Kowalski, ${ }^{1,2}$ Liang Su, ${ }^{1,2}$ Jarrod D. Milshtein, ${ }^{1,3}$ Fikile R. Brushett ${ }^{1,2}$ \\ ${ }^{1}$ Joint Center for Energy Storage Research \\ ${ }^{2}$ Department of Chemical Engineering, Massachusetts Institute of Technology \\ ${ }^{3}$ Department of Materials Science \& Engineering, Massachusetts Institute of Technology
}

\begin{abstract}
:
Cost-effective electrochemical energy storage will play a critical role as society transitions to a sustainable energy economy. Nonaqueous flow batteries employing redox active organic molecules are an emerging energy storage concept. A key advantage of this device over the more established aqueous flow battery is the promise for higher cell potentials ( $>3 \mathrm{~V}$ ), enabled by the larger electrochemical stability windows of nonaqueous electrolytes. Additionally, nonaqueous flow batteries could leverage new redox couples that are incompatible with aqueous electrolytes due to low solubility, chemical reactivity, and redox potentials outside of the aqueous stability window. Taking advantage of these characteristics may lead to higher energy densities, smaller system footprints, and lower costs. This mini review summarizes recent developments in allorganic nonaqueous chemistries with a focus on tailoring organic molecules for improved physical and electrochemical properties. Key opportunities and challenges in the science and engineering of these devices are presented with a goal of meeting stringent grid cost targets.
\end{abstract}

Keywords: redox flow batteries; nonaqueous; organic compounds; molecular engineering 


\section{Introduction}

Fossil fuel technologies comprise the backbone of the global energy economy, where the transportation and electric power sectors are the two largest consumers. The abundance, availability, and affordability of liquid fossil fuels have been a key driver of industrialization over the past century. In the future, this energy mix will not be feasible because the rising population and continuing economic growth, particularly in the developing world, are projected to increase the global energy demand and consumption by $48 \%$ by 2040 [1]. Greenhouse gas emissions and air pollution from fossil fuel combustion are also of growing concern [2]. The past 20 years has seen a renaissance of renewable electricity generators (e.g., solar photovoltaic, wind, tidal), where dramatic cost reductions, coupled with government-incentivized policies, have enabled market penetration at the utility and residential scale [3]. The intermittent nature of renewable generation, however, presents an additional challenge to the electric power sector, which is already seeking to modernize its infrastructure to improve efficiency, reduce environmental impact, and enhance resiliency [4,5].

Electrochemical energy storage will play a critical role as society transitions to a sustainable energy economy [6,7]. Redox flow batteries (RFBs) are rechargeable electrochemical devices that store energy in flowable solutions or suspensions of electroactive species housed in external tanks and pumped to a power-converting electro-reactor (Figure 1). As compared to enclosed batteries (e.g., lithium-ion, lead-acid), the RFB architecture offers several advantages including the ability to decouple the power rating (reactor size) from the energy capacity (tank size), a high ratio of active-to-inactive materials, simplified manufacturing, a longer service life with full charge/discharge cycles, and improved safety. Due to their low energy density and integrated 
design requirements, RFBs are best suited for megawatt-scale energy storage with long charge/discharge durations ( 4-5 hours).

Since the 1970s, researchers have explored numerous flow battery chemistries including iron-chromium, bromine-polysulfide, vanadium-polyhalide, and all-vanadium systems [8]. Several studies have pursued hybrid systems, in which one or both electrode reactions involve a deposition/dissolution process, such as the zinc-bromine and soluble lead acid systems. While some of these RFB chemistries have been demonstrated at scale, none has experienced widespread commercial success due to a number of technical and economic challenges [8]. Recent dramatic advances in renewable electricity generation have spurred research and development targeting lower costs and improved performance for RFB technologies. This renewed interest has also led to a number of informative review papers and perspectives on the technical and scientific aspects of these devices $[9,10]$.

Recently, organic molecules have been examined as redox active species in nonaqueous (NAq) RFBs. Organics offer several advantages over typical transition metal salts.

- Organic molecules are typically comprised of earth-abundant elements, and thus, their cost and availability is less constrained by the production and reserves of rarer elements. Moreover, transition metals must be mined and purified from ore, but organics can conceivably be synthesized in a sustainable fashion using green chemistry routes.

- While the known periodic table restricts the number of inorganic redox couples, a broader array of redox-active organic molecules is available, allowing for the realization of new redox couples.

- Modification of redox moieties, or the surrounding molecular structure, can tailor key electrochemical (e.g., redox potential) and physical properties (e.g., solubility). In addition, a 
number of organics are capable of multiple electron transfer events, which could lead to higher charge storage capacities and, subsequently, higher cell energy densities.

Transitioning from aqueous (Aq) to NAq electrolytes offers the opportunity to increase cell voltage via wider electrochemical stability windows of the NAq electrolytes, typically 3-4 V depending on salt, solvent, and electrode material [11]. Additionally, new electrochemical couples which are incompatible with aqueous electrolytes due to low solubility, chemical reactivity, or redox potentials outside of the stability window can now be considered. Moreover, a diversity of supporting salts and molecular solvents exist, which can be tailored to a specific organic redox chemistry. If realized together, these benefits can lead to higher energy densities, smaller system footprints, and lower costs of energy storage [12]. While organic NAqRFBs are a relatively infant technology in comparison to their aqueous counterparts, organic NAqRFBs represent a burgeoning research area, rich with scientific and engineering opportunities. Here, we describe recent advances in the developments of redox active organic molecules for all-organic flow batteries and highlight key challenges and opportunities for the research community. 


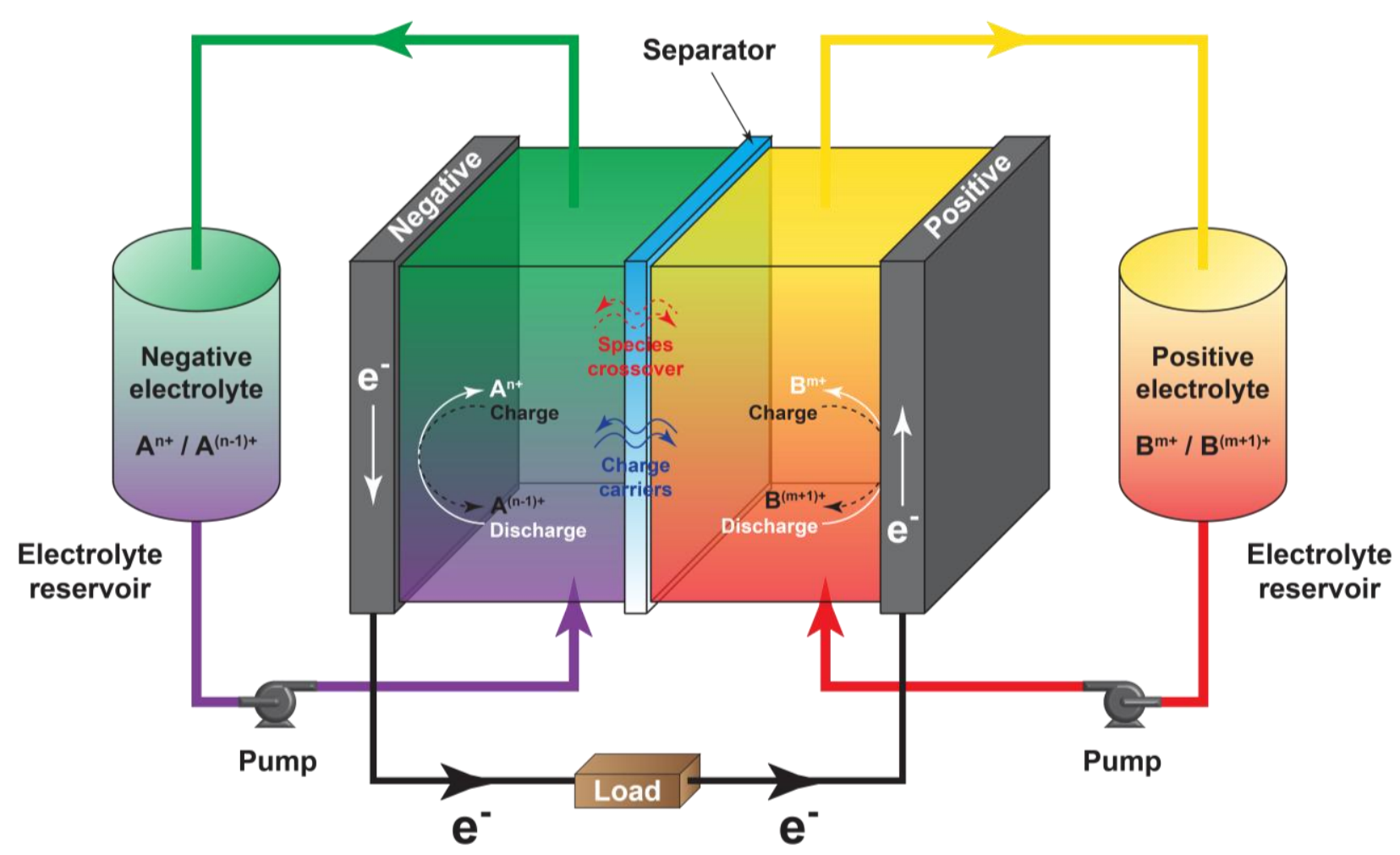

Figure 1: Redox flow battery schematic. The system includes electrochemical reactors, storage vessels, circulation pumps, a heat exchanger, and power conditioning equipment. During operation, the positive and negative electrolytes are fed to one or more electrochemical reactors, where the active species are oxidized or reduced to charge or discharge the battery. In the reactor, the electrolytes are separated by a membrane or porous separator, which facilitates transport of charge carrying ions while blocking active species crossover. In this schematic, the cell is discharging. This figure is adapted with permission from the Journal of the Electrochemical Society [10].

\section{All-Organic NAqRFB Redox Couples}

Though first conceived in the 1980s, early reports of NAqRFBs were limited and redox chemistries were based on metal or metal-ligand coordination complexes (e.g., $\left.R u(b p y)_{3}\left[\mathrm{BF}_{4}\right]_{2}\right)$. In 2011, Li et al. reported the first NAqRFB employing all-organic active materials, where 2,2,6,6-tetramethyl-1-piperidinyloxy (TEMPO) and N-methylphthalimide (NMP) served as the positive (high potential) and negative (low potential) active materials, respectively, exhibiting a cell potential of $1.6 \mathrm{~V}$ [13]. After that initial report, several organic redox couples have been paired together as proof-of-concept energy storage systems in demonstration cells utilizing coin 
cells, Swagelok cells, H-cells, or flow cells. Figure 2 highlights some prominent redox chemistries, illustrating the active species pairs and supporting salts.

For high potential materials, many of the initial demonstrations employ active compounds that were originally designed as redox shuttles for overcharge protection in lithium ion batteries (e.g., dialkoxybenzenes, phenothiazines, nitroxides [14]). Although the desirable molecular properties differ for RFB applications, overcharge protection materials have proven performance and durability in relatively harsh electrochemical environments and thus are a reasonable starting point for RFBs. A few key examples are TEMPO [13], 4-Oxo-TEMPO (O-TEMPO) [15], 2,5di-tert-butyl-1,4-bis(2-methoxyethoxy)benzene (DBBB) [16], 2,5-di-tertbutyl-1-methoxy-4-[2'methoxyethoxy]benzene (DBMMB) [17], and 3,7-bis(trifluoromethyl)-N-ethylphenothiazine (BCF3EPT) [18].

For low potential materials, active compounds have been more difficult to identify due to the lack of research for other applications, leading to the exploration of new redox active organic molecules such as 2,3,6-trimethylquinoxaline (TMQ) [16,18], 9-fluorenone (FL) [17], and (1S)(+)-camphoroquinone (CPQ) [15]. In two instances, symmetric organic NAqRFB prototypes utilized the same parent organic molecule as both the high and low potential active materials. These demonstration chemistries are 2-phenyl-4,4,5,5-tetramethylimidazoline-1-oxyl-3-oxide (PTIO) [19] and 1,4-bis(isopropylamino)anthraquinone (Disperse Blue 134, DB134) [20]. Such symmetric organic chemistries are especially attractive because the fully discharged cell contains the same molecule, in the same oxidation state, on both sides of the battery, unlike other symmetric flow batteries (e.g. all-vanadium) that access different redox states [20]. Discharging to a single parent species helps alleviate deleterious RFB cycling effects such as irreversible crossover and electrolyte imbalance [20,21]. Of the published set of symmetric demonstration 
chemistries, DB134 is particularly notable because this molecule has five accessible redox states and can engage in two electron transfer events on both the high and low potential sides of the battery [20]. In addition, several studies have proposed other families of small redox active organic molecules, notably rubrene [22], cyanoazacarbons [23], biphenyl [24], and octafluoronaphthalene [24], for implementation in NAqRFBs, but, at the time of this review, none of these classes of molecules have been demonstrated in a prototype cell of any kind.

Recently, redox active organic macromolecules, such as polymers, have been investigated, beginning with an insoluble suspension of polythiophene (PTP) in a symmetric cell [25]. This concept not only illustrated polymers as active materials for flow batteries but also suggests that the organic species must not necessarily be soluble in the electrolyte. The first soluble organic redox active polymer (RAP) proposed for use in an RFB was poly(vinylbenzyl ethyl viologens) in 2014 [26], and the first implementation of a full NAq cell based on soluble organic polymers arrived two years later in the form of poly(boron-dipyrromethane) (BODIPY) in a symmetric configuration [27]. Soluble RAPs show promise for enabling low-cost size exclusion separators, in place of more expensive charge-selective membranes, but currently suffer from high solution viscosities, which lead to additional pumping and reactor design challenges [26].

While these initial studies focus on proof-of-concept demonstrations of energy storage promise, the proposed redox chemistries are unlikely to supplant state-of-the-art AqRFBs, as:

- The redox chemistries are generally between $1.5-2.5 \mathrm{~V}$, on par with aqueous zinc-bromine and lead-acid systems, and therefore, do not leverage the full range of the electrochemical stability window enabled by non-aqueous electrolytes. 
- While the possibility of high active species concentration is discussed in a few reports, most demonstration cells operate at concentrations from 0.02 to $0.5 \mathrm{M}$, which, in combination with the moderate voltage, leads to a relatively low cell energy density $\left(0.5-15 \mathrm{Wh} \mathrm{L}^{-1}\right)$

- Reported cycle lifetimes are typically less than 100 cycles, often with noticeable capacity decay. With the exception of TEMPO, which has been proven to be highly stable under certain electrolyte conditions $[28,29]$, the lifetimes are about an order of magnitude too short. Some of these challenges are exacerbated by limitations in prototype cell engineering, which hinder high concentration and high current density cycling of organic active materials. To date, the highest current densities and concentrations displayed were $20 \mathrm{~mA} \mathrm{~cm}^{-2}$ and $0.5 \mathrm{M}$, respectively, by Duan et al. [19], which are both insufficient to compete with state-of-the-art AqRFBs [21]. Efforts have thus shifted towards tailoring redox active organic molecules to realize desired properties for NAq RFBs.
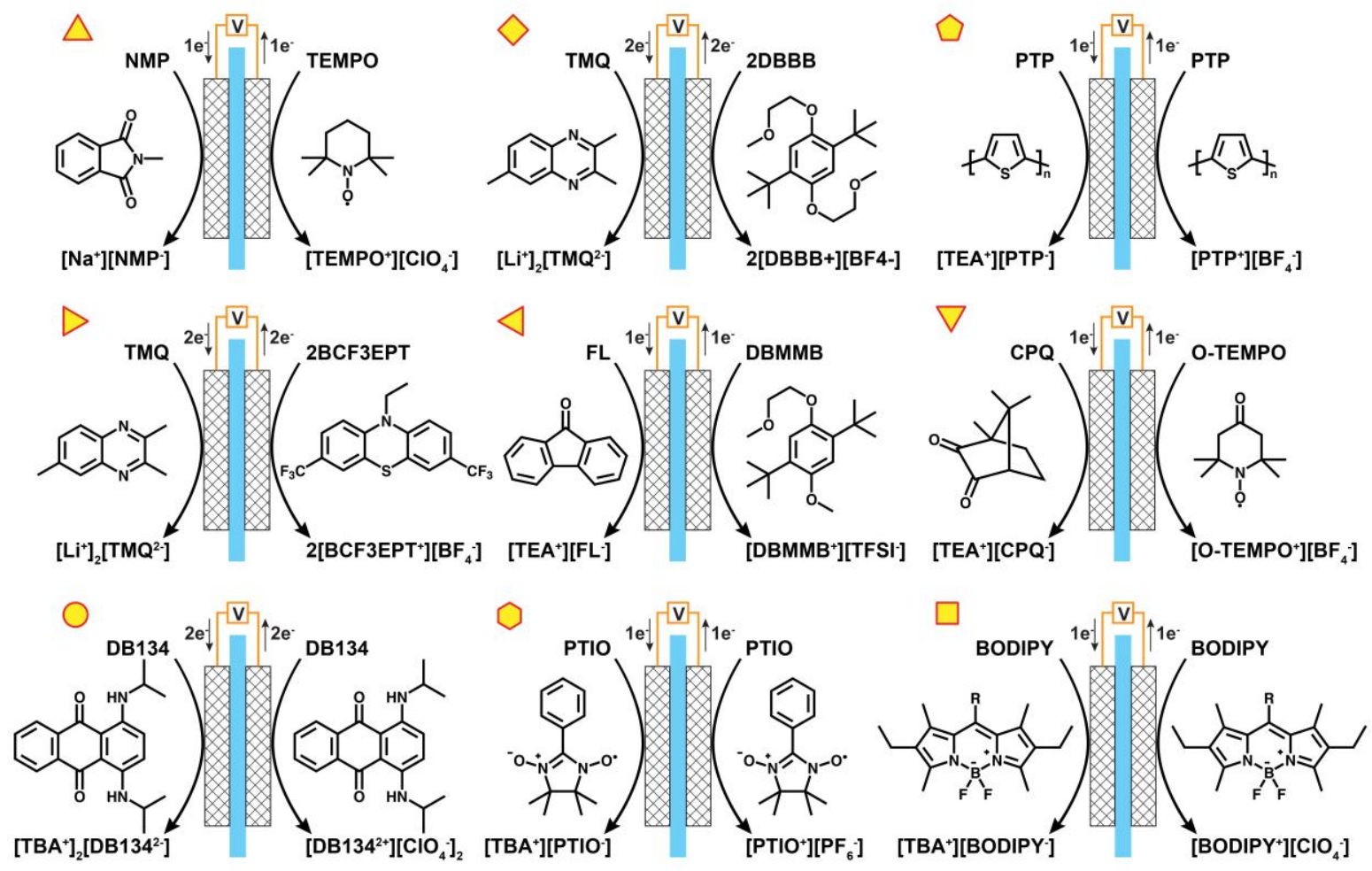
Figure 2: Summary of organic NAq chemistries demonstrated in coin cell, Swagelok cell, Hcell, or flow cell configurations [13,15-20,25,27].

A

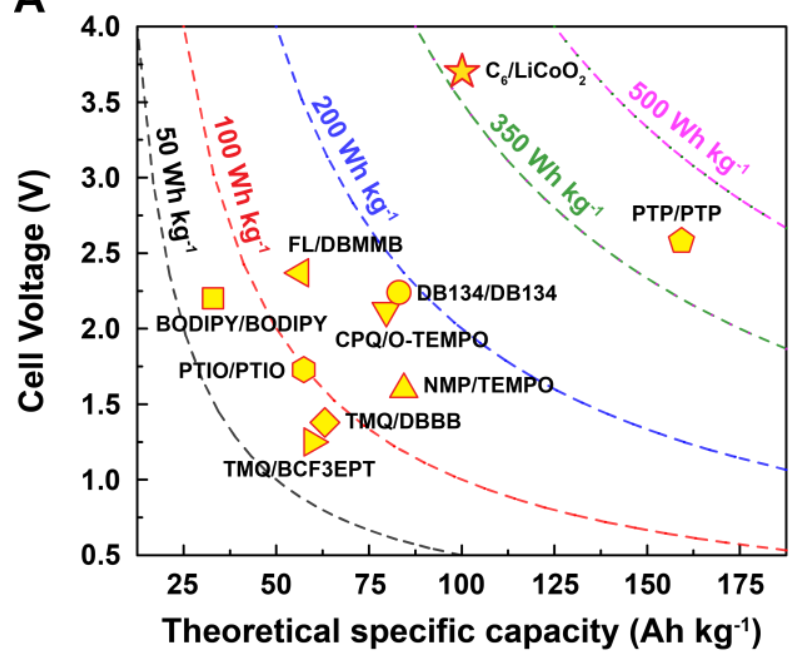

B

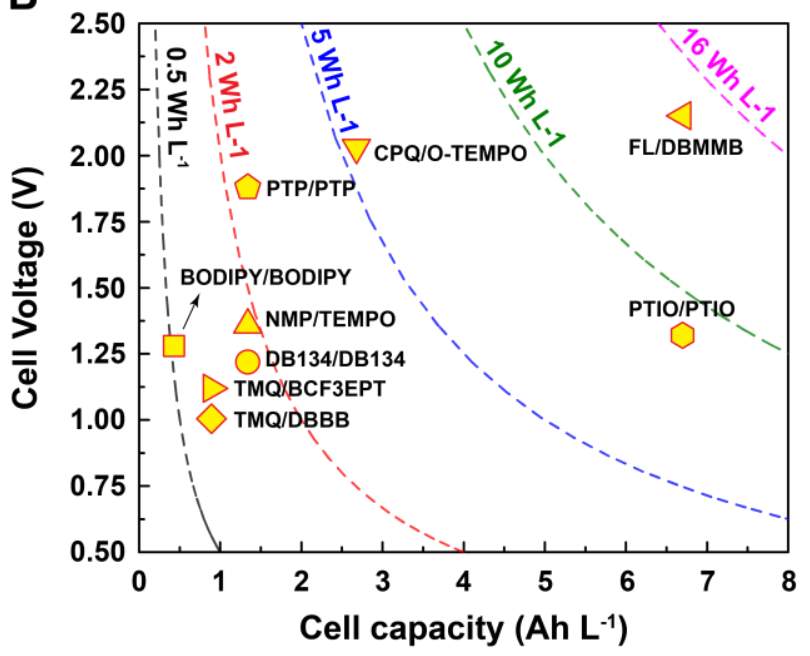

Figure 3. The theoretical capacity vs. the cell voltage for the NAqRFB chemistries shown in Figure 2. (A) The theoretical specific capacities are calculated based on the molecular weight of the active species, which provide the limiting capacity of the proposed chemistries. The cell voltages are calculated based on the experimentally determined half-wave potentials using cyclic voltammetry for each. The broken line for each color represents the relationship between capacity and voltage at the corresponding specific energy. (B) The cell capacities are calculated based on the active species concentration used in the corresponding reference. The cell voltages are the experimentally determined average cell discharge voltage. The broken line for each color represents the relationship between capacity and voltage at the corresponding energy density. 


\section{Molecular engineering of active materials}

Molecular engineering is the process of functionalizing a redox active moiety to obtain desired properties in line with performance and cost targets. The ideal redox active organic molecule would have a redox potential near the upper or lower edge of the electrolyte stability window, a high solubility, long-term stability through all states-of-charge in relevant electrolyte solutions, and a low molecular weight to maximize charge storage capacity and minimize chemical costs. Increasing active species solubility raises the maximum feasible active species concentration, which can decrease solvent costs, increase energy density, and lower kinetic or mass transfer resistances[30]. Additionally, decreasing molecular weight improves the active species gravimetric capacity, decreasing active species cost per unit charge stored. Further, highly stable active species can improve device lifetime, and extreme redox potentials can enable greater cell potentials, yielding higher energy and power densities. Optimizing these factors within the available design space of organic NAqRFBs could lead to a long lasting, energy dense, and low cost system [12]. Here we review several initial molecular engineering campaigns with a focus on designing to one or more of the aforementioned traits.

As previously mentioned, redox shuttles for overcharge protection materials in lithium-ion batteries serve as a good starting point for new high-potential organic active materials because they exhibit many desirable electrochemical and physical properties, including high solubility, facile kinetics, good stability, and a high redox potential. DBBB has served as a platform for several design efforts involving substituted dialkoxybenzenes (Figure 4) because of its success as a redox shuttle and early demonstration in all-organic NAqRFBs [16]. Huang et al. targeted increased solubility and reduced molecular weight through the development of DBBB derivatives with asymmetric structure through modification of the relative lengths of ether chains 
appended to the oxygen leading to a series of liquid-phase active materials [31]. The asymmetry in the structures causes the molecule to have a larger overall dipole, which leads to a more polar molecule and therefore increases the solubility in polar solvents. In a parallel study, Huang and Su et al. targeted reduced stable structures through a subtractive engineering approach which sought to remove or replace bulky functional groups on the DBBB structure that did not contribute to charge storage [32]. As shown in Figure 4, without modifying the electrochemically active center (bold, red) of DBBB, the substitution of $t$-butyl groups by methyl groups and the shortening of the methoxyethyl groups significantly decrease the molecular weight and thereby improve the intrinsic capacity of the methoxybenzene-based redox active species [32]. The 2,5-dimethyl-1,4-dimethoxybenzene showed similar behavior to the parent DBBB compound. This indicates that the methyl groups were still sufficient to provide enough steric hindrance to protect the radical once the material is charged and the shortened ether chain still provided enough electron delocalization to stabilize the radical cation.
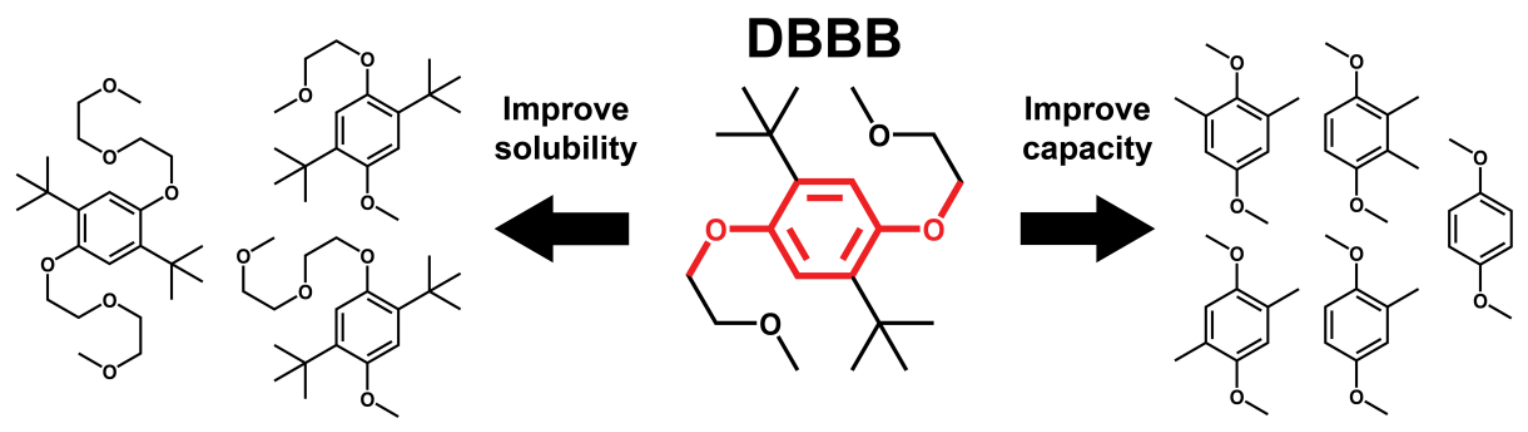

Figure 4: Modifications to the core structure of DBBB for improved properties [31,32]

Similarly, phenothiazine derivatives, also originally examined for overcharge protection [33,34], are now under investigation for NAqRFB applications with early efforts focused on increasing the redox potential through the addition of electron withdrawing groups. When 
electron withdrawing groups (e.g. halides, a nitro- group, cyanide group) are added to a redox moiety, the valence electrons are more tightly bound to the core structure. Therefore, more energy is necessary to remove an electron, and the redox potential, which is related to the ionization energy, is increased. Kaur et al. targeted a higher redox potential by adding trifluoromethyl groups to phenothiazine, boosting the redox potential by $320 \mathrm{mV}$ [18].

Turning toward the negative electrolyte, quinoxaline [16,35], pyridine [36], and anthraquinone [37] derivatives have been the most well-studied materials. Quinoxaline has been the focus of low potential compounds since its initial appearance in the TMQ/DBBB all-organic NAqRFB [16]. In this seminal study, structure - activity relationships were identified by investigating the effect of the number and position of the substituents (methyl groups and phenyl groups) on the benzene and pyrazine rings. To enable the high-throughput selection of a quinoxaline-based negative compound, Assary et al. calculated the reduction potential of 40 quinoxaline derivatives using quantum chemical methods [35]. Moreover, it has been recently discovered that Lewis acids such as $\mathrm{BF}_{3}$, can promote the electrochemical activity of this category of compound by forming a $\mathrm{BF}_{3}$ - complex with the lone pair on nitrogen atoms [38,39]. This has shown to be a valuable tool in the development of other negative compounds [36] for NAqRFBs.

Using pyridine as the starting material, Sevov et al. described an iterative workflow of molecular engineering for the development of low potential compounds [36]. The work first examined the decomposition pathways for pyridine derivatives and then modified the derivatives further to block the degradation pathway selectively. The final product, N-methyl 4acetylpyridinium tetrafluoroborate, shows high solubility (1.6 M in acetonitrile), low molecular weight $\left(222 \mathrm{~g} \mathrm{~mol}^{-1}\right)$, two reversible electron transfer processes on the cyclic voltammetry 
timescale (in the presence of $\mathrm{LiBF}_{4}$ ), and decent store stability (2 months) of both charged and discharged states. Of course, further characterization of this compound in battery cycling experiments is necessary to validate its promise as a negative compound in NAqRFB.

Anthraquinone derivatives showed recent promise as a low potential compound in aqueous RFBs [40]. Modifications by Wang et al. have adapted the base anthraquinone molecule for NAq electrolytes by adding ether chains to increase solubility [37]. Two ether chains were added to the anthraquinone base molecule in the 1 and 5 positions. The flexible ether chains add to the overall polarity of the molecule and increase the disorder when the molecule dissolves. Both of these properties contribute to increasing the solubility in polar solvents.

The compounds discussed here represent just a few of the available starting materials for molecular engineering in the broad design space of organic redox active compounds. The same molecular engineering techniques and criteria can apply to other classes of organic compounds proposed for energy storage applications [41-44].

\section{Conclusions \& Outlook}

Electrochemical energy storage systems will play a pivotal role in the widespread integration of renewable, non-dispatchable energy sources and in the improvement of energy efficiency of the electric power sector. RFBs are particularly attractive for these applications due to their favorable combination of performance, cost, and safety. However, present storage technologies are not yet cost-competitive, which drives research and development efforts towards new redox chemistries, electrolyte formulations, and cell designs. NAqRFBs utilizing redox active organic materials are a nascent but emerging storage concept with the potential for achieving the low costs needed for economic viability. In 2014, Darling et al. highlighted performance metrics required to realize a $\$ 100 / \mathrm{kWh}$ NAqRFB, indicating that while such a battery was feasible, 
significant improvements are required over the current demonstrations [12]. These benchmarks present an opportunity for target-guided fundamental research of novel NAq redox chemistries including:

- Identifying electrochemical couples that, when paired together, enable a $3 \mathrm{~V}$ cell potential or higher. Multi-electron transfer couples would increase charge storage capacity and alleviate concentration requirements.

- Enhancing the stability of organic molecules, particularly the charged radicals, in NAq electrolytes to enable 1000's of cycles with minimal capacity fade. Electrolyte selection has already been shown to impact the stability of redox species and therefore contributes to a delicate balance between achieving required concentrations and reversibility [17,39].

- Increasing the solubility of organic molecules in NAq electrolytes at all states-of-charge. The high concentration regime, where solvent and solute molecules are of the same order of magnitude, is relatively unexplored, and may require the development of new analytical methods to quantify solution structure and electrochemical performance.

Given the interconnected nature of these requirements, coupled with the multi-dimensional design space afforded by organic molecules, high throughout computation tools, in combination with targeted experimental work are a powerful strategy for driving discovery and development $[36,45,46]$. In concert with these molecular efforts, advances in the engineering science of NAq RFBs are needed:

- Tailoring rheological and electrochemical properties of redox electrolytes to minimize pumping costs and maximize charge transfer rates.

- Quantifying selectivity of porous electrode materials, particularly at potentials near the electrochemical stability window to minimize electrolyte decomposition. 
- Designing stable, selective, and conductive membranes to prevent species crossover.

- Constructing research prototypes, inspired by more mature electrochemical technologies (e.g., fuel cells), that enable high current densities and high active species concentrations. - Predicting the chemical costs of newly-developed organic molecules in a more rigorous fashion.

Opportunities exist for significant improvements through engineering of organic molecules for NAqRFBs. This multidisciplinary challenge requires expertise and collaboration across multiple fields including electrochemical science, molecular modeling and synthesis, cell engineering, and techno-economic assessment. Together with an evolving regulatory environment, these R\&D activities are expected to lead to the deployment of a range of new cost-effective flowable energy storage systems. 


\section{Acknowledgements:}

This work was funded by the Joint Center for Energy Storage Research (JCESR), an Energy Innovation Hub funded by the United States Department of Energy, Office of Science, Basic Energy Sciences. The authors thank John Barton and Thomas Carney for insightful discussion and constructive feedback. Jarrod Milshtein gratefully acknowledges the National Science Foundation Graduate Research Fellowship Program for additional financial support (DGE 1256260) 


\section{References}

1. U.S. Energy Information Administration: International Energy Outlook 2016.

2. Stocker TF, Qin D, Plattner G-K, Tignor MMB, Allen SK, Boschung J, Nauels A, Xia Y, Bex V, Midgley PM: Climate change 2013: the physical science basis. Climate change 2013: the physical science basis. 2013.

3. MIT Energy Initiative: The Future of Solar Energy. 2015.

4. U.S. Department of Energy: Grid Energy Storage December 2013.

5.**Yang Z, Zhang J, Kintner-Meyer MCW, Lu X, Choi D, Lemmon JP, Liu J: Electrochemical Energy Storage for Green Grid. Chem. Rev. 2011, 111:3577-3613.

(A high-level review article discussing different electrochemical energy systems for grid-scale energy storage)

6. Chu S, Majumdar A: Opportunities and challenges for a sustainable energy future. Nature 2012, 488:294-303.

7. Dunn B, Kamath H, Tarascon J-M: Electrical Energy Storage for the Grid: A Battery of Choices. Science 2011, 334:928-935.

8. Su L, Kowalski JA, Carroll KJ, Brushett FR: Recent Developments and Trends in Redox Flow Batteries. In Rechargeable Batteries. Edited by Zhang Z, Zhang SS. Springer International Publishing; 2015:673-712.

9. Soloveichik GL: Flow Batteries: Current Status and Trends. Chem. Rev. 2015, 115:11533-11558.

10. Perry ML, Weber AZ: Advanced Redox-Flow Batteries: A Perspective. J. Electrochem. Soc. 2016, 163:A5064-A5067.

11. Xu K: Electrolytes and Interphases in Li-Ion Batteries and Beyond. Chem. Rev. 2014, 114:11503-11618.

12.**Darling RM, Gallagher KG, Kowalski JA, Ha S, Brushett FR: Pathways to low-cost electrochemical energy storage: a comparison of aqueous and nonaqueous flow batteries. Energy Environ. Sci. 2014, 7:3459-3477.

(Techno-economic model describing the necessary parameters that must be met to reach economically feasible flow batteries for both aqueous and nonaqueous systems. A set of material targets for the electrolyte, active materials, and reactor are outlined. The economically accessible design space is shown, and nonaqueous systems have promise.)

13. Li Z, Li S, Liu S, Huang K, Fang D, Wang F, Peng S: Electrochemical Properties of an AllOrganic Redox Flow Battery Using 2,2,6,6-Tetramethyl-1-Piperidinyloxy and NMethylphthalimide. Electrochem. Solid-State Lett. 2011, 14:A171-A173.

14. Chen Z, Qin Y, Amine K: Redox shuttles for safer lithium-ion batteries. Electrochimica Acta 2009, 54:5605-5613. 
15. Park S-K, Shim J, Yang J, Shin K-H, Jin C-S, Lee BS, Lee Y-S, Jeon J-D: Electrochemical properties of a non-aqueous redox battery with all-organic redox couples. Electrochem. Commun. 2015, 59:68-71.

16.**Bushett FR, Vaughey JT, Jansen AN: An All-Organic Non-aqueous Lithium-Ion Redox Flow Battery. Adv. Energy Mater. 2012, 2:1390-1396.

(Proof-of-concept nonaqueous lithium-ion redox flow battery using completely organic active materials. The high potential and low potential organic molecules used were 2,5-di-tert-butyl-1,4bis(2-methoxyethoxy)benzene and 2,3,6-trimethyl quinoxaline respectively.)

17.*Wei X, Xu W, Huang J, Zhang L, Walter E, Lawrence C, Vijayakumar M, Henderson WA, Liu T, Cosimbescu L, et al.: Radical Compatibility with Nonaqueous Electrolytes and Its Impact on an All-Organic Redox Flow Battery. Angew. Chem. Int. Ed. 2015, 54:8684-8687.

(The effect of the electrolyte on the cycling stability for the 9-fluorenone and 2,5-di-tert-bytul-1methoxy-4-[2'-methoxyethoxy]benzene flow cell system was examined. Five different electrolytes were examined and were shown to have different coulombic, voltage, and energy efficiencies.)

18. Kaur AP, Holubowitch NE, Ergun S, Elliott CF, Odom SA: A Highly Soluble Organic Catholyte for Non-Aqueous Redox Flow Batteries. Energy Technol. 2015, 3:476-480.

19. Duan W, Vemuri RS, Milshtein JD, Laramie S, Dmello RD, Huang J, Zhang L, Hu D, Vijayakumar $\mathrm{M}$, Wang $\mathrm{W}$, et al.: A symmetric organic-based nonaqueous redox flow battery and its state of charge diagnostics by FTIR. J. Mater. Chem. A 2016, 4:5448-5456.

20. Potash RA, McKone JR, Conte S, Abruña HD: On the Benefits of a Symmetric Redox Flow Battery. J. Electrochem. Soc. 2016, 163:A338-A344.

21. Darling R, Gallagher K, Xie W, Su L, Brushett F: Transport Property Requirements for Flow Battery Separators. J. Electrochem. Soc. 2016, 163:A5029-A5040.

22. Chakrabarti MH, Dryfe RAW, Roberts EPL: Evaluation of electrolytes for redox flow battery applications. Electrochimica Acta 2007, 52:2189-2195.

23. Rasmussen PG: Electrical storage device utilizing pyrazine-based cyanoazacarbons and polymers derived therefrom. Patent US8080327 B1, 2011.

24. Gong K, Fang Q, Gu S, Li SFY, Yan Y: Nonaqueous redox-flow batteries: organic solvents, supporting electrolytes, and redox pairs. Energy Environ. Sci. 2015, 8:3515-3530.

25. Oh SH, Lee C-W, Chun DH, Jeon J-D, Shim J, Shin KH, Yang JH: A metal-free and all-organic redox flow battery with polythiophene as the electroactive species. J. Mater. Chem. A 2014, 2:19994-19998.

26. Nagarjuna G, Hui J, Cheng KJ, Lichtenstein T, Shen M, Moore JS, Rodríguez-López J: Impact of redox-active polymer molecular weight on the electrochemical properties and transport across porous separators in nonaqueous solvents. J. Am. Chem. Soc. 2014, 136:16309-16316.

27. Winsberg J, Hagemann T, Muench S, Friebe C, Häupler B, Janoschka T, Morgenstern S, Hager MD, Schubert US: Poly(boron-dipyrromethene)—A Redox-Active Polymer Class for Polymer RedoxFlow Batteries. Chem. Mater. 2016, doi:10.1021/acs.chemmater.6b00640. 
28. Wei X, Xu W, Vijayakumar M, Cosimbescu L, Liu T, Sprenkle V, Wang W: TEMPO-Based Catholyte for High-Energy Density Nonaqueous Redox Flow Batteries. Adv. Mater. 2014, 26:7649-7653.

29. Milshtein JD, Barton JL, Darling RM, Brushett FR: 4-acetamido-2,2,6,6-tetramethylpiperidine-1oxyl as a model organic redox active compound for nonaqueous flow batteries. J. Power Sources 2016, 327:151-159.

30. Alotto P, Guarnieri M, Moro F: Redox flow batteries for the storage of renewable energy: A review. Renew. Sust. Energ. Rev. 2014, 29:325-335.

31. Huang J, Cheng L, Assary RS, Wang P, Xue Z, Burrell AK, Curtiss LA, Zhang L: Liquid Catholyte Molecules for Nonaqueous Redox Flow Batteries. Adv. Energy Mater. 2015, 5:n/a-n/a.

32.*Huang J, Su L, Kowalski JA, Barton JL, Ferrandon M, Burrell AK, Brushett FR, Zhang L: A subtractive approach to molecular engineering of dimethoxybenzene-based redox materials for non-aqueous flow batteries. J. Mater. Chem. A 2015, 3:14971-14976.

(Modified the 2,5-di-tert-butyl-1,4-bis(2-methoxyethoxy)benzene base structure by subtractive engineering by reducing the size of the polyethylene oxide chains and the tert-butyl groups. One of these molecular weight reductions doubled the capacity without dramatically affecting the redox potential or stability.)

33. Narayana KA, Casselman MD, Elliott CF, Ergun S, Parkin SR, Risko C, Odom SA: N-Substituted Phenothiazine Derivatives: How the Stability of the Neutral and Radical Cation Forms Affects Overcharge Performance in Lithium-Ion Batteries. Chemphyschem Eur. J. Chem. Phys. Phys. Chem. 2015, 16:1179-1189.

34. Kaur AP, Ergun S, Elliott CF, Odom SA: 3,7-Bis(trifluoromethyl)-N-ethylphenothiazine: a redox shuttle with extensive overcharge protection in lithium-ion batteries. J. Mater. Chem. A 2014, 2:18190-18193.

35. Assary RS, Brushett FR, Curtiss LA: Reduction potential predictions of some aromatic nitrogencontaining molecules. RSC Adv. 2014, 4:57442-57451.

36.*Sevov CS, Brooner REM, Chénard E, Assary RS, Moore JS, Rodríguez-López J, Sanford MS: Evolutionary Design of Low Molecular Weight Organic Anolyte Materials for Applications in Nonaqueous Redox Flow Batteries. J. Am. Chem. Soc. 2015, 137:14465-14472.

(Molecular modifications were made to the pyridine structure to improve the stability based on possible decomposition pathways. The molecular design lead to a more stable low potential material with a low molecular weight.)

37. Wang W, Xu W, Cosimbescu L, Choi D, Li L, Yang Z: Anthraquinone with tailored structure for a nonaqueous metal-organic redox flow battery. Chem. Commun. 2012, 48:6669-6671.

38. Chénard E, Sutrisno A, Zhu L, Assary RS, Kowalski JA, Barton JL, Bertke JA, Gray DL, Brushett FR, Curtiss LA, et al.: Synthesis of Pyridine- and Pyrazine-BF ${ }_{3}$ Complexes and Their Characterization in Solution and Solid State. J. Phys. Chem. C 2016, 120:8461-8471.

39. Carino EV, Diesendruck CE, Moore JS, Curtiss LA, Assary RS, Brushett FR: BF 3 -promoted electrochemical properties of quinoxaline in propylene carbonate. $R S C A d v$. 2015, 5:1882218831. 
40. Huskinson B, Marshak MP, Suh C, Er S, Gerhardt MR, Galvin CJ, Chen X, Aspuru-Guzik A, Gordon RG, Aziz MJ: A metal-free organic-inorganic aqueous flow battery. Nature 2014, 505:195-198.

41. Song Z, Zhou H: Towards sustainable and versatile energy storage devices: an overview of organic electrode materials. Energy Environ. Sci. 2013, 6:2280-2301.

42. Connelly NG, Geiger WE: Chemical Redox Agents for Organometallic Chemistry. Chem. Rev. 1996, 96:877-910.

43. Boeré RT, Roemmele TL: Electrochemistry of redox-active Group 15/16 heterocyles. Coord. Chem. Rev. 2000, 210:369-445.

44. Liang Y, Tao Z, Chen J: Organic Electrode Materials for Rechargeable Lithium Batteries. Adv. Energy Mater. 2012, 2:742-769.

45. Cheng L, Assary RS, Qu X, Jain A, Ong SP, Rajput NN, Persson K, Curtiss LA: Accelerating Electrolyte Discovery for Energy Storage with High-Throughput Screening. J. Phys. Chem. Lett. 2015, 6:283-291.

46. Su L, Ferrandon M, Kowalski JA, Vaughey JT, Brushett FR: Electrolyte Development for NonAqueous Redox Flow Batteries Using a High-Throughput Screening Platform. J. Electrochem. Soc. 2014, 161:A1905-A1914. 